\title{
Biliary Obstruction
}

National Cancer Institute

\section{Source}

National Cancer Institute. Biliary Obstruction. NCI Thesaurus. Code C60698.

Blockage in the biliary tract that carries bile from the liver to the gallbladder and small intestine. Causes include gallstones, biliary tract strictures and inflammation, pancreatitis, cirrhosis, lymph node enlargement, and bile duct and pancreas neoplasms. 